\title{
Causes of defaulting from antiretroviral therapy and their prevention: a qualitative study of adherent clients and defaulters in Nigeria
}

\author{
Olayinka Stephen Ilesanmi ${ }^{1,2}$, Aanuoluwapo Adeyimika Afolabi ${ }^{1}$ \\ ${ }^{1}$ Department of Community Medicine, College of Medicine, University of Ibadan, Ibadan, Oyo State, Nigeria \\ ${ }^{2}$ Department of Community Medicine, University College Hospital, Ibadan, Oyo State, Nigeria
}

\begin{abstract}
Introduction: In Nigeria, non-adherence to treatment or its interruption endanger successful human immunodeficiency virus (HIV) control. This study identified the causes and prevention of defaulting from antiretroviral therapy (ART) among adults on ART at Federal Medical Centre, Owo, Ondo State, Nigeria.

Material and methods: To explore the perspectives, thoughts, behaviors of respondents, and new ideas, qualitative information was collected using focus group discussions (FGD) guide and in-depth interviews (IDI) guide. IDI of defaulters and FGD with persons compliant with treatment were conducted to identify the barriers to retention in care and its prevention. Data were transcribed and analyzed using the thematic framework approach.

Results: Reasons for defaulting included traveling out of place of residence, lack of support from employer, failure to disclose HIV status to partner and caregivers. Both FGD and IDI revealed poverty/ unemployment, pill fatigue, religious factors, lack of family support, family problems, and stigmatization/discrimination, as the major reasons for defaults. Results from the FGD suggested strengthening of counselling sessions and improved follow-up to reduce defaulting.

Conclusions: Encouraging clients to access care in health facilities, which are near their places of residence will reduce the cost of care and improve the treatment and care. Introduction of Saturday and Sunday clinic days, with options of evening visits in the evening, will promote adherence to treatment.
\end{abstract}

HIV AIDS Rev 2021; 20, 2: 136-143 DOI: https://doi.org/10.5114/hivar.2021.107240

Key words: HIV, antiretroviral treatment, stigmatization, disclosure, defaulting from antiretroviral therapy.

\section{Introduction}

The attendance of scheduled clinic visits is crucial in the treatment of people living with human immunodeficiency virus (HIV)/acquired immunodeficiency syndrome (AIDS) (PLWHA) [1]. In particular, missing clinic appointments has

been shown to reduce adherence to antiretroviral therapy (ART) [2]. High level of adherence to ART is necessary in the prevention of virologic failure and development of positive health outcomes [2]. An adherence rate of $95 \%$ is recommended for patients to ensure viral suppression and optimal outcomes of treatment [2]. Consequences of defaulting from

Article history:

Received: 09.09.2020

Received in revised form: 18.10.2020

Accepted: 11.12.2020

Available online: 30.06 .2021
International Journal of HIV-Related Problems

HIV \& AIDS

R e vi e w 
HIV treatment include inadequate health outcomes and poor quality of life. Moreover, there is an increased disease burden on defaulting patients.

To address defaulting of clinic visits and high rate of treatment failure, adherence counselling has been recommended [3]. This is because patients who attend clinic visits are not likely to skip counselling sections [4]. Attending counselling sessions would enable PLWHA to benefit maximally from ART program. Results of a systematic review of studies conducted in sub-Saharan Africa revealed that up to two years on ART resulted to an increased retention in care by up to $14 \%$ [5]. The advantages of retention in care of patients on ART include combined clinical and laboratory monitoring, adherence support through private and public health facilities, and community [5]. Retention in ART care is also important for the enhancement of ART decentralization efforts by the government, and the intensification of time spent on the first-line regimen results in retention in care, which reduces the risk of treatment failure [5].

\section{Health-related}

Reasons for missing clinic appointments are not fully known in Nigeria. In a study conducted in Port-Harcourt, Nigeria, mothers who brought their children for pediatric ART reported avoiding clinic visits [6]. Factors associated with missed clinic appointments among HIV-infected adult patients include delayed access to health facilities, illicit drug use, HIV stigma, mental health disorders, perceived low social support, younger age, presence of comorbidities, and less severe illness [7]. Moreover, dissatisfaction with healthcare providers have also been linked to appointments' missing $[7,8]$. An evidence suggests that retention in ART programs is dependent on some factors. These can be conveniently classified as socio-economic, provider-related, client-related, and healthcare system factors. Each of these factors or their interaction can directly affect retention in care [9].

Evaluation of context-specific factors affecting participation in clinic visits is important to reduce the risk of poor outcomes, complications, and opportunistic infections among PLWHA. Adherence studies have always tended to address issues of patients who have been retained in program rather than patients were lost to the program $[10,11]$. Research on attendance and non-attendance of ART clinic visits would help to recommend appropriate interventions, which could further improve the quality of care of PLWHA in Ondo State and other parts of Nigeria. Therefore, here, we present the findings from a qualitative study among patients attending and those defaulting from ART clinic visits.

\section{Material and methods}

\section{Study area}

The study was conducted in Owo, an ancient city located in Ondo State, Southwest Nigeria. The Federal Medical Cen- tre (FMC), Owo, offers services in all clinical specialties, and laboratory, radiology, and social/ welfare as found in other teaching hospitals. It also offers residency training programs in family medicine, surgery, medicine, obstetrics and gynecology, and pediatrics.

\section{Study setting}

The ART clinic of the FMC, Owo, was established in February 2006 by the Family Health International and Global HIV/AIDS Initiative in Nigeria (FHI/GHAIN). The FMC, Owo offers comprehensive HIV/AIDS treatment and care. People registered in the ART center are usually individuals diagnosed with HIV after voluntary counselling and testing (VCT). Pregnant women also join the ART clinic after completing antenatal clinic, where the knowledge on prevention of mother-to-child transmission (PMTCT) is conveyed.

\section{Study design}

A qualitative study using in-depth interviews (IDI) and focus group discussions (FGD) was conducted. IDIs were held with ART defaulters in the ART clinic of FMC, while FGDs were conducted among patients who were currently on ART.

\section{Study population}

The population for this study included HIV-positive clients who were aged $>18$, were receiving ART, but had defaulted from care. Other members of the study population involved HIV-positive individuals on medications, who were still on ART. Defaulters were those who had missed at least two clinic appointments and ART drug pickup in the last year before the study. Data of defaulters were obtained from their clinical records and used for tracing. Tracing started with phone calls to invite defaulters to the hospital. Defaulters who were not willing to come to the hospital were visited at their residence. On the other hand, compliant patients were individuals who had been on ART for at least one year and had attended all scheduled clinic visits.

\section{Study instruments}

To explore the perspectives, thoughts, behavior of the respondents, and new ideas, qualitative information was collected with FGD guide and IDI guide.

FGDs formation: In total, four FGDs were carried out, including FGD 1 with younger adult males aged 18-34 years; FGD 2 with older males aged 35 years and above; FGD 3 with younger adult females aged 18-34 years; and FGD 4 with older females aged 35 years and above.

Each focus group consisted of 7 to 10 persons, excluding the moderator, recorder, and observer. The moderator facilitated the discussion using the FGD guide, and ensured participation of every member of the group. Discussions were 
recorded by a recorder, with the aid of a digital recorder and by taking notes on paper, while the observer took note of the non-verbal expressions of the participants. Each FGD occurred on clinic days in one of the offices in the hospital. The FGD guide was developed using probe questions reviewed by experts.

Each IDI was conducted one-on-one, with a interviewee and a recorder. The interviewer used the IDI guide, which was developed using experts' reviewed probe questions. Probing statements, such as "Could you explain further..." and "Could you be more specific...", were used to obtain more information from the respondents. The discussion was recorded with a digital recorder as well as taking notes on paper. The IDI took place within the hospital premises. The main issues discussed in FGDs and IDIs were experiences with taking ART, knowledge of HIV treatment among PLWHA enrolled on ART, and how defaulting from ART can be prevented.

\section{Data collection}

Data were collected over one month. FGDs took place within the hospital buildings. IDIs also took place within the hospital premises and at homes of defaulters, who did not return to the hospital after being contacted on the phone. Interviews were conducted in either English or Yoruba languages. The note-taker/ recorder and the interviewer were trained by the principal investigator prior to data collection.

\section{Data management}

Qualitative data were recorded and analyzed using thematic framework approach for qualitative data analysis. This was an iterative process of analysis, which started immediately after the first interview and continued throughout the research. A thematic framework was developed from emerging themes in all the interviews. This was done after each interview to enhance subsequent interviews. As themes emerged, they were indexed and compared with themes from subsequent interviews, until a saturation point was achieved. The results were then presented in quotes, boxes, and diagrams for comparability purposes.

\section{Ethical considerations}

Ethical approval was obtained from the Federal Medical Centre, Owo, Health Research Ethics Committee (FMC/ OW/380/VOL.XXIX/ 07). The permission to conduct the study was obtained from the coordinator of HIV/AIDS treatment and care center.

\section{Results}

Overall, four IDIs were carried out, including IDI 1 with younger adult males, aged 18-34 years; IDI 2 comprising older males, aged 35 years and above; IDI 3 involving young- er adult females, aged 18-34 years; and IDI 4 with older females, aged 35 years and above.

The results of the qualitative investigation of factors associated with default among adults on ART program in Ondo State are presented in the following sub-section.

\section{Perception of treatment requirements}

Both defaulters and non-defaulters appreciated the necessity of ART. They both consented to getting tired of using drugs, as presented below.

\section{Defaulters}

A younger male defaulter said that "Using the drug is tiring, the start date is known, but no one knows when to stop it". An older female said "[ART] is not available in small hospitals found everywhere. I have to come here to collect the drugs". However, a younger female defaulter said that the drugs help her in gaining strength; however, leaving her work to come and assess the treatment is not always possible. The same older female defaulter said: “...though side effect are often experienced, stopping ART has more negative effects, though, feeling of wellness and strength give the feeling that the drug is not needed" (F, IDI 4).

\section{Non-defaulters}

A younger non-defaulter said that "Living depends on the drugs" and an older female also added: "Only the use of our medication guarantees good health, use of the drugs is therefore compulsory".

"Treatment is easier when drugs are fewer, using many drugs continuously to stay healthy is not easy (just saying the truth)" (M, IDI 1).

\section{Respondents' knowledge on HIV treatment requirement}

The respondents' knowledge about HIV treatment differed between defaulters and non-defaulters. Both believed HIV treatment should not be stopped, as demonstrated below.

\section{Defaulters}

Some defaulters declared that HIV drugs should be stopped only when other drugs (non-HIV-related) are to be used.

\section{Non-defaulters}

Some of the views were as follows:

"...number of tablets and timing of use should not be changed without doctor's advice" (F, FGD 2).

"...the drug can only work optimally when it is used daily without any interruption; those who used their drugs daily are less likely to be sick" (M, FGD 3). 


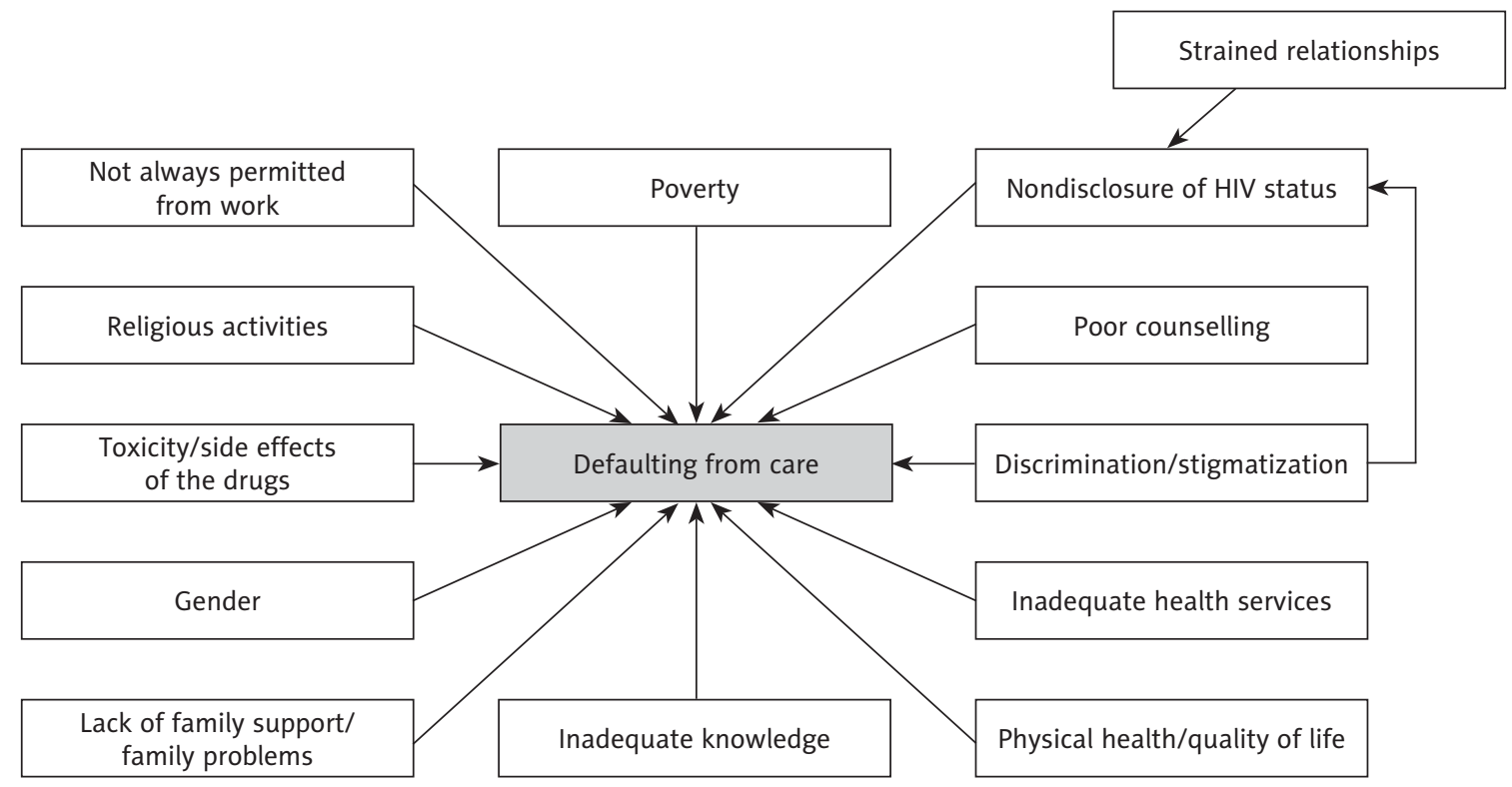

Figure 1. Respondents' views about factors associated with defaulting from care

\section{Respondents' reasons for defaulting}

Respondents from both categories described factors that could make a patient on medication to default from care. Frequent appointments and inability to get away from work were mentioned. In addition, the issues of clinic not opening on Saturdays and lack of transportation were the main reasons for default. Numerous reasons were highlighted by participants during the FGDs and IDIs as barriers to clinic appointments among PLWHA receiving care at Federal Medical Centre, Owo. These are summarized in Figure 1.

\section{Poverty/unemployment}

Poverty, especially resulting from unemployment or under-employment, was described as one of the major factors affecting respondents' compliance with clinic visits. Difficulty in securing employment due to poor health was also linked to failure to attend clinic visits. Lack of job leads to insufficient amount of money for food and transport fare. Quotes from respondents are below:

\section{Defaulters}

"No job opportunity exists; small salary for the job I do may not be paid as when its due. This affects planning for hospital visitation" (F, IDI 4).

"If there is no food to eat, all attention is drawn to getting food and it can distract from visiting the hospital" (M, IDI 3).

"...lack of job opportunity will make one not to be able to attend the hospital, as necessary" (M, IDI 1).
A younger female added: “...lack of adequate money to come to the hospital prevents me from coming as scheduled".

\section{Non-defaulters}

Non defaulters also submitted reasons for possible default from treatment.

"Unemployment can lead to poor financial state, thereby, contributing to poverty and failure to continue with clinic attendance" (M, FGD 3).

"Income or salary can contribute to regular clinic visit because, if one is unemployed, lack of money can result as defaulting from care" (F, FGD 2).

Younger male non-defaulters felt not having money can only affect transport when the cost is high: "...poverty, i.e., not having money, can hinder clinic visits, especially when transport fare is high" (M, FGD 1).

\section{Physical health and quality of life}

Good physical health was described by respondents in all FGDs as an important criterion for not defaulting from care. Seeking care continuously also leads to good quality of health. Quality of life affects day-to-day activities, including the ability to visit the hospital. These viewpoints are presented in Table 1.

\section{Lack of family support/family problems}

Family disharmony, death of spouse, and lack of care giver (especially among the defaulters) were other import- 
Table 1. Respondents' perception about relationship between physical health, quality of life, and defaulting from care

\begin{tabular}{|c|c|}
\hline Defaulters & Non-defaulters \\
\hline $\begin{array}{l}\text { "...if a person is sick, there won't be adequate energy and strength to } \\
\text { come to the hospital" } \\
\qquad(\mathrm{F}, \mathrm{IDI} 2)\end{array}$ & \multirow{2}{*}{$\begin{array}{c}\text { "...Good physical health will improve quality of life } \\
\text { and help us not to default from care, we need } \\
\text { strength to be able to come to the hospital } \\
\text { when necessary" } \\
\text { (F, FGD 2) }\end{array}$} \\
\hline $\begin{array}{l}\text { "...those with suboptimal level of health are expected } \\
\text { to visit the hospital more regularly for them to regain their strength" } \\
\qquad(\mathrm{M}, \mathrm{IDI} 1)\end{array}$ & \\
\hline $\begin{array}{l}\text { "Physical health will affect the quality of life in many ways } \\
\text { only the drugs when properly used will result in good health" } \\
\text { (M, IDI 3) }\end{array}$ & \multirow{2}{*}{$\begin{array}{l}\text { "...if anything is wrong with the physical health, } \\
\text { it will definitely affect the quality of life, this } \\
\text { can make the person to be admitted and visit } \\
\text { the hospital more frequently" } \\
\text { (M, FGD 3) }\end{array}$} \\
\hline $\begin{array}{l}\text { "If there is poor health, it will definitely affect attendance at the clinic } \\
\text { because thinking about health condition causes distractions..." } \\
\text { (F, IDI 4) }\end{array}$ & \\
\hline
\end{tabular}

Table 2. Respondents' recommendations to the hospital

$\left.\begin{array}{c|c}\hline \text { Defaulters } & \text { Non-defaulters } \\ \hline \begin{array}{c}\text { to come to clinic" } \\ \text { (M, IDI 3) }\end{array} & \begin{array}{c}\text { "...if the hospital can provide special counselling for } \\ \text { defaulters it will help them to remember they need } \\ \text { to be consistent" } \\ \text { (M, FGD 3) }\end{array} \\ \hline \text { "...sending confidential text messages will serve as a reminder" } \\ \text { (M, IDI 2) }\end{array}\right)$

ant factors identified as affecting regular clinic attendance of respondents. The following quotes support this opinion.

\section{Defaulters}

A younger female defaulter said: "I have 3 children with their father dead; when their father was sick, we spent money on him before he died. I have to struggle and send the children to school; all this stress affected regular clinic attendance". An older male added: “...in my own case, my wife's family accused me of killing their child; they felt I did not take care of her when she was sick and died; all these have been disturbing my peace and the ability to take good care of myself". The same older male non-defaulter agreed to the problem of family support and suggested a solution: "...problems of lack of family support can result in defaulting from care and this can be solved by letting people around us to understand our problems, and let them know that we need their support".

\section{Religious/spiritual factors}

As shown in the quotes below, involvement in religious activities is one main factor identified by both defaulters and non-defaulting respondents affecting retention in care/defaulting.

\section{Defaulters}

A younger female felt: "When you have Christ, you have inner strength to go about your daily duties".

\section{Non-defaulters}

An adult male non-defaulter said that a patient may default if he/she thinks that prayer has worked and he/she is no longer positive. Non-defaulters in their own words: “...religious understanding can also affect patient's attendance in the hospital; some of our colleagues stopped coming to the hospital after being prayed for. They come back later with complications" they added.

\section{Discrimination and stigmatization}

The issue of discrimination and stigmatization affecting regular clinic attendance resulted from IDI with female defaulters. This was reportedly one of the reasons for defaulting. This problem was not reported among the non-defaulters.

\section{Defaulters}

A younger female felt: "Being HIV-positive usually make other people to look down on us; we are at the mercy of healthcare workers to receive drugs and care". Whilst an older female 
also said: "Most of us look sick; other patients in the hospital can easily identify us with our status; they usually see us as a wayward person to have HIV. Reducing contact with the hospital will prevent us from being looked down upon".

\section{Recommendations from respondents on how defaulting from care can be reduced}

Below are some recommendations from the respondents for the hospital management and PLWHA towards reducing defaulting from care among those on treatment.

\section{Hospital}

The participating defaulters suggested that the hospital should introduce weekend appointments, and that more should be done, including giving transport fare to them. However, the non-defaulters suggested increasing clinic appointments to every 4 months, and the use of reminders, like phone call, at least once a week before the appointment date. Some quotes are presented below.

\section{People living with HIV/AIDS}

To reduce defaulting from care, the defaulters suggested they needed to encourage themselves and use reminders not to forget appointments, while the non-defaulters recommended a better awareness of HIV/ AIDS in the community, which was said to reduce stigmatization. An adult male defaulter suggested they should "...assist each other by contributing money when there is lack". An adult female added that: "We must be reminded at frequent intervals of our appointments; text messages and phone calls can be used". An older female group advised that PLWHA should make good use of the opportunity that they have. "The drugs, if they were to be sold, many of us will not be able to afford it; why don't we come and take free drugs when due". An older male non-defaulter also added: "We must be our brother's keeper; we can call them and visit them when they are not seen in the clinic".

\section{Discussion}

In the present study, we found that poverty, strained relationships, poor counselling, and lack of family support were some reasons emphasized by participants for defaulting from ART care. ART was greatly acknowledged by patients as a means of staying healthy, even in their HIV-positive state. Even though the patients appreciated the need for ART, they reported pill fatigue. Adverse effects of medication, pill burden, and feeling unwell were frequently expressed as important reasons for defaulting from care. Adverse effects and pill burden of ARV drugs have been identified to compromise the effectiveness of HIV treatment programs, particularly in low- and middle-income countries $[12,13]$. Interestingly, the respondents stated that HIV treatment should not be stopped, as improved health after taking treatment was re- ported as a facilitator of adherence. Nevertheless, ART programs should increase the availability of regimens with fewer adverse reactions and reviewed number of pills, wherever possible.

Respondents reported that poor counselling affected treatment adherence. Literature demonstrated that good relationship with patient and taking time to address concerns can improve adherence as well as interventions, such as counselling, education, and peer support [14, 15]. HIV patients are less likely to skip ART appointments when they have a good knowledge about the etiology of the disease, and understand that ART is effective with strict adherence [16]. Therefore, healthcare workers, who are involved in HIV treatment, need to increase counselling modalities. Here, we also found that closure of HIV clinics on weekends could discourage ART attendance, especially when patients are engaged all week at work. This emphasize the need for enhanced capacity for HIV clinics to be able to receive patients not only on weekdays, but also on weekends.

Consistent with previously published research, poverty resulting from unemployment, under-employment, or cost associated with care, affected patients' adherence to HIV treatment. This finding is corroborated by findings from Ekiti State and Port-Harcourt, Nigeria $[6,17]$. One major barrier to adherence in all the studies examined was the cost. Although the ARV drugs are heavily subsidized in the Nigerian program, patients have to bear the cost of transportation to and from the health facility as well as the cost of laboratory testing, which can be a huge burden for some patients. The IDIs with defaulters revealed that a major reason for defaulting was traveling beyond the residential area. This was confirmed in another study conducted in Southwest Nigeria [22]. Therefore, poverty is a strong determinant of nonadherence; although, several specific factors that interplay with poverty may also contribute to poor adherence.

Defaulting from care hinders adherence, and defaulting from ART care has been reported as a major predictor of viral suppression of HIV replication, emergence of ART drug resistance, disease progressions, and death $[19,20]$. Therefore, HIV treatment adherence monitoring is an essential public health surveillance tool in the prevention of HIV in Nigeria [21]. Educational and programmatic efforts are needed to improve treatment awareness and access for HIV-infected persons in both public and private care settings. Interventions to address this challenge should be holistic, including government and health facilities levels, crucially designed, with full participation of PLWHA.

Stigma and discrimination continue to be an issue in HIV programing, occurring at several levels [18]. Defaulting patients reported this issue to be a major factor affecting their compliance to the treatment regimen. Several studies have reported that fear of stigmatization caused patients to hide their ART or skip doses when in the presence of others, especially those to whom they have not disclosed their status. In addition, self-stigma may lead patients to be unwilling or fearful to take medicines in other people presence. 
Defaulting from treatment was associated with non-disclosure of HIV status to partner. The participants who did not disclose their status to their partners were less likely to receive support to maintain an appointment. One of the main reasons for disclosing HIV status to partner was to ensure that a trusted person could assist in seeking care in the event of an illness. Stigma and rejection have been documented as a common experience of PLWHA, especially in developing countries $[22,23]$. For many PLWHA, stigma is a barrier to disclose their status, and to get access to available support and care services $[22,23]$. In addition, partners of HIVpositive patients encourage them to go for treatment and drug refill. Non-disclosure and lack of home-based support continue to be a critical barrier for maintenance and adherence to ART programs [24]. Moreover, lack of support from workplace prevented some defaulters, and non-disclosure of HIV-positive status to family members was also found to be a cause for ART default among PLWHA in Ethiopia [25].

\section{Limitations}

Different interviews methods used for both groups could have influenced comparisons and conclusions drawn from the study. Despite these limitations, the results obtained contribute immensely to the current body of knowledge.

\section{Conclusions}

ART interventions are beneficial in improving the quality of life of HIV-positive individuals. Although, the benefits of ART are known among HIV patients, certain delimiting factors could influence defaulting from HIV care. Therefore, we recommend improved decentralization of HIV care clinics to places, where a need for such exists. Also, HIV-positive persons should be encouraged to register for ART services at health facilities within their residential areas to overcome the challenge associated with frequent hospital visitations and high costs of accessing care. Capacity enlargement of health facilities and workers is required, so that patients who are available for ART care on weekends would not be denied their right to quality care. Health workers should develop problem-solving approaches to identify challenges, which prompt patients to default from HIV care. Supports from peer groups, health workers, family members, and community members are required to prevent stigmatization, and encourage adherence to antiretroviral therapies and hospital attendance as scheduled.

\section{Conflict of interest}

The authors declare no conflict of interest with respect to the research, authorship, and/or publication of this article.

\section{References}

1. Sileo KM, Wanyenze RK, Kizito W, et al. Multi-level determinants of clinic attendance and antiretroviral treatment adherence among fishermen living with HIV/AIDS in communities on Lake Victoria, Uganda. AIDS Behav 2019; 23: 406-417.

2. Erlwanger AS, Joseph J, Gotora T, et al. Patterns of HIV care clinic attendance and adherence to antiretroviral therapy among pregnant and breastfeeding women living with HIV in the context of option B+ in Zimbabwe. J Acquir Immune Defic Syndr 2017; 75 : S198-S206.

3. Jiamsakul A, Kerr SJ, Kiertiburanakul S, et al.; TREAT Asia HIV Observational Database (TAHOD). Early suboptimal ART adherence was associated with missed clinical visits in HIV-infected patients in Asia. AIDS Care 2018; 30: 1560-1566.

4. Gokarn A, Narkhede MG, Pardeshi GS, Doibale MK. Adherence to retroviral therapy. J Assoc Physicians India 2012; 60: 16-20.

5. Umeokonkwo CD, Onoka CA, Agu PA, Ossai EN, Balogun MS, Ogbonnaya NU. Retention in care and adherence to HIV and AIDS treatment in Anambra State Nigeria. BMC Infect Dis 2019; 19: 654.

6. Ugwu R, Eneh A. Factors influencing adherence to paediatric antiretroviral therapy in Portharcourt, South-South Nigeria. Pan Afr Med J 2013; 16: 30.

7. Ngandu NK, Jackson D, Lombard C, et al. Factors associated with non-attendance at scheduled infant follow-up visits in an observational cohort of HIV-exposed infants in South Africa, 2012-2014. BMC Infect Dis 2019; 19 (Suppl 1): 788.

8. DiCarlo A, Fayorsey R, Syengo M, et al. Lay health worker experiences administering a multi-level combination intervention to improve PMTCT retention. BMC Health Serv Res 2018; 18: 17.

9. Abuogi LL, Smith C, McFarland EJ. Retention of HIV-infected children in the first 12 months of anti-retroviral therapy and predictors of attrition in resource limited settings: a systematic review. PLoS One 2016; 11: e0156506.

10. Ketema AK, Weret ZS. Assessment of adherence to highly active antiretroviral therapy and associated factors among people living with HIV at Debrebrihan Referral Hospital and Health Center, northeast Ethiopia: a cross-sectional study. HIV/AIDS (Auckl) 2015; 7: 75-81.

11. Reda AA, Biadgilign S. Determinants of adherence to antiretroviral therapy among HIV-infected patients in Africa. AIDS Res Treat 2012; 2012: 574656.

12. AIDS Info. Guidelines for the Use of Antiretroviral Agents in Adults and Adolescents with HIV. US Department of Health and Human Services. 2019. Available from: https://aidsinfo.nih.gov/guidelines/ html/1/adult-and-adolescent-arv/31/adverse-effects-of-arv (Accessed: 13.08.2020).

13. Li H, Marley G, Ma W, et al. The role of ARV associated adverse drug reactions in influencing adherence among HIV-infected individuals: a systematic review and qualitative meta-synthesis. AIDS Behav 2017; 21: 341-351.

14. Fox MP, Paascoe S, Huber AN, et al. Adherence clubs and decentralized medication delivery to support patient retention and sustained viral suppression in care: results from a cluster-randomized evaluation of differentiated ART delivery models in South Africa. PLoS Med 2019; 16: e1002874.

15. Agarwal R, Rewari BB, Allam RR, Chava N, Rathore AS. Quality and effectiveness of counselling at antiretroviral therapy centers in India: capturing counsellor and beneficiary perspectives. Int Health 2019; 11: 480-486.

16. Zhang Y, Xu G, Hou J, et al. Problem-based learning could tackle the issue of insufficient education and adherence in people living with HIV/AIDS. Front Pharmacol 2019; 10: 901.

17. Adeoti AO, Dada M, Elebiyo T, Fadare J, Ojo O. Survey of antiretroviral therapy adherence and predictors of poor adherence among HIV patients in a tertiary institution in Nigeria. Pan Afr Med J 2019; 33: 277.

18. Chambers LA, Rueda S, Baker DN, et al. Stigma, HIV, and health: a qualitative synthesis. BMC Public Health 2015; 15: 848.

19. Barabona G, Mahiti M, Masoud S, et al. Pre-treatment and acquired HIV drug resistance in Dar es Salaam, Tanzania in the era of teno- 
fovir and routine viral load monitoring. J Antimicrob Chemother 2019; 74: 3016-3020.

20. Ndubuka NO, Lim HJ, van der Wal DM, Ehlers VJ. Health-related quality of life of antiretroviral treatment defaulters in Botswana. South Afr J HIV Med 2016; 17: 475.

21. Meloni ST, Agbaji O, Chang CA, et al. The role of point-of-care viral load monitoring in achieving the target of $90 \%$ suppression in HIV-infected patients in Nigeria: study protocol for a randomized controlled trial. BMC Infect Dis 2019; 19: 368.

22. Aransiola J, Imoyera W, Olowookere S, Zarowsky C. Living well with HIV in Nigeria? Stigma and survival challenges preventing optimum benefit from an ART clinic. Glob Health Promot 2014; 21: 13-22.

23. Odimegwu C, Adedini SA, Ononokpono DN. HIV/AIDS stigma and utilization of voluntary counselling and testing in Nigeria. BMC Public Health 2013; 13: 465.

24. Kalomo EN. Associations between HIV-related stigma, self-esteem, social support, and depressive symptoms in Namibia. Aging Ment Health 2018; 22: 1570-1576.

25. Gadisa T, Tymejczyk O, Kulkarni SG, et al. Disclosure history among persons initiating antiretroviral treatment at six HIV clinics in Oromia, Ethiopia, 2012-2013. AIDS Behav 2017; 21: 70-81. 